\title{
Arsenic Resistance Gene Expression and Microbial Diversity from Altered Irrigated Paddy Soil in Bengal Delta
}

ARNAB MAJUMDAR ${ }^{1 *}$, MUNISH KUMAR UPADHYAY ${ }^{3}$, ASHISH KUMAR SRIVASTAVA ${ }^{2}$, SUDHAKAR SRIVASTAVA ${ }^{3}$ , MANOJ KUMAR JAISWAL ${ }^{1}$, SUTAPA BOSE ${ }^{1}$

${ }^{1}$ Department of Earth Sciences, IISER Kolkata, Mohanpur, West Bengal, India-741246 (*Correspondancearnabmajumdar891@gmail.com;)

${ }^{2}$ Nuclear Agriculture and Biotechnology Division, BARC, Mumbai, Maharashtra, India-400085

${ }^{3}$ Plant Stress Biology Laboratoy, IESD, BHU, Varanasi, Uttar Pradesh, India-221005

\section{Rationale}

Agricultural soil has varied physic-chemical influences and nutritional variability that plays crucial role in microbial diversity. This study revealed the relation between altered irrigation and soil arsenic (As) flux that induces microbial resistance towards As and differential gene expression.

\section{Procedures followed}

Soil physico-chemical parameters ( $\mathrm{pH}$, ORP, TDS, OM, Conductivity, microbial biomass), were measured along with soil nutritional assessment. Determination of elemental concentrations has been validated in soil-plant samples using WD-XRF, and Synchrotron XRF by fusion bead and pressed bead method. Large scale paddy fields were designed as conventional flooding (CF) and intermittent flooding (IF) irrigation practices considering seasonal variation in wintery and monsoonal cultivation. Below are the proposed parametric equations-

$A d C_{s}{ }^{a 2}=A h^{a} C_{W^{2}}+A d C_{s}{ }^{a 1}+W^{a} C_{r}{ }^{a}$ and $\mathbf{C}_{w}{ }^{2}\left(D_{P}\right)=\left[A_{s} C_{s}{ }^{b 1}+A^{b}{ }^{b} C_{w}{ }^{1}\right]-\left[A_{d C} C^{b 2}+A\left(h^{b}-h^{a}\right) C_{w}{ }^{1}\right]$

Those differentially irrigated soil were analysed for total microbial diversity by metagenomics as well as arsenic resistance gene dependence microbial community profiling. A control field soil was also checked for metagenomics to identify any occurrence of irrigation-affected gene activity on indigenous microbes. Real-time FE-SEM imaging was done to observe the changes in the internal vascular system in rice plants under these two irrigations and bacterial abundance.

\section{Results in brief}

As flux has been determined from the proposed two equations depending on seasonal variation and metagenomics from altered irrigated soil has shown to have an effect on arsenic registance gene expression on microbes and its diversity.

[1] Zhang et al., (2019), Sci. Total Environ. 687, 790-799. [2] Majumdar et al., (2019), Ecotoxicol. Environ. Safety. 180, 770-789. 